\title{
WEAK FAMILIES OF MAPS
}

\author{
J. C. Taylor
}

(received March 12, 1965)

1. Introduction. Let $\Omega$ be an index set and for each $\alpha \in \Omega$ let $\overline{\mathrm{f}_{\alpha}: \mathrm{X} \rightarrow \mathrm{X}_{\alpha}}$ be a function where $\mathrm{X}$ and $\mathrm{X}_{\alpha}$ are sets. Assume that, for each $\alpha$, a topology $\underline{\mathrm{O}}_{\alpha}$ is given for $\mathrm{X}_{\alpha}$. Then, as is well-known, the functions $f_{\alpha}$ and the topologies $\underline{O}_{\alpha}$ determine a topology for $\mathrm{X}$. This is the so-called weak or initial topology, which is generated by $\bigcup_{\alpha}\left\{f_{\alpha}^{-1}\right.$ OlO $\left.O \underline{O}_{\alpha}\right\}$.

Bourbaki [1] shows that the weak topology is the unique topology $O$ for $X$ satisfying the following condition: a function $f: Y \rightarrow X$ is $(\underline{T}, \underline{O})$-continuous if and only if, for each $\alpha$, $f_{\alpha} \circ f$ is $\left(\underline{T}, \underline{O}_{\alpha}\right)-$ continuous. This suggests that the concept of a weak topology could be defined using the language of category: theory.

Let $A$ denote the category of topological spaces, and let $E$ denote the category of sets. Denote by a : $A \rightarrow B, b, \ldots$ the morphisms of $A$ and by $f: X \rightarrow Y, g, \ldots$ those of $\underline{E}$. Let $F: \underline{A} \rightarrow \underline{E}$ denote the forgetful functor.

Denote by $A$ the space $(X, O)$, by $A_{\alpha}$ the space $\left(\mathrm{X}_{\alpha}, \underline{\mathrm{O}}_{\alpha}\right)$, and let $\left(\mathrm{f}_{\alpha}\right)$ be a family of functions $\mathrm{f}_{\alpha}: \mathrm{X} \rightarrow \mathrm{X}_{\alpha}$. The topology of the space $A$ is the weak topology determined by $\left(f_{\alpha}\right)$ and $\left(A_{\alpha}\right)$ if and only if the following assertion holds. A function $f: Y \rightarrow X$ is of the form $F(b)$, for $b: B \rightarrow A$, if and only if, for each $\alpha$, there exists $b_{\alpha}: B \rightarrow A_{\alpha}$ with $f_{\alpha} \circ f=F(b)$.

Canad. Math. Bull. vol. 8, no. 6, 1965 
Assume that the topology of $A$ is the weak topology determined by $\left(f_{\alpha}\right)$ and $\left(A_{\alpha}\right)$. Since the identity map ${ }^{1} X$ is $F\left(1_{A}\right)$, each $f_{\alpha}$ is of the form $F\left(a_{\alpha}\right)$. Hence, as is well known, the family $\left(f_{\alpha}\right)$ determines a family $\left(a_{\alpha}\right)$ of morphisms of $\underline{A}$.

The family $\left(a_{\alpha}\right)$ of morphisms a of $\underline{A}$, with common domain $A$, has the following property: if a function $f: F(B) \rightarrow F(A)$ is such that there exists a family $\left(b_{\alpha}\right)$ of morphisms $b_{\alpha}: B \rightarrow A_{\alpha}$ with, for each $\alpha, F\left(a_{\alpha}\right) \circ f=F\left(b_{\alpha}\right)$, then there exists a unique $b: B \rightarrow A$ with $F(b)=f$ and, for each $\alpha, a_{\alpha} \circ b=b_{\alpha}$. In terms of diagrams, ( $\left.a_{\alpha}\right)$ is such that the commutativity, for each $\alpha$, of

$$
F(B)
$$

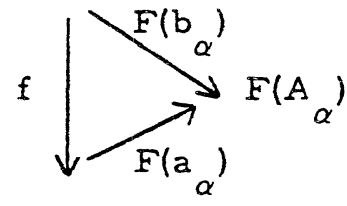

$F(A)$

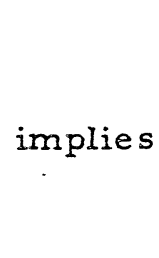

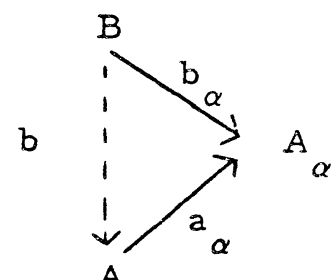

A

with $F(b)=f$.

Let $\underline{A}$ and $E$ be arbitrary categories, and let

$F: \underline{A} \rightarrow \underline{E}$ be a covariant functor. The purpose of this expository note is to provide some examples and to discuss some elementary properties of families $\left(a_{\alpha}\right)$ of morphisms $a_{\alpha}$ of $A$ which have the above property. When $\underline{E}$ is the trivial category with a unique morphism such families define the direct products that exist in A.

While the theory outlined here is essentially a translation of Bourbaki's theory of initial structures [2] into the language of categories, it differs in several respects. The emphasis here is on families of morphisms of $A$, rather than on the determination of an object of $A$ by families of morphisms of $E$ and families of objects of $\bar{A}$. Further, the theory of initial structures restricts $A^{-}$to be the category determined by a type 
of structure, $E$ to be the category of sets, and $F$ to be the forgetful functor.

In the case where the index set is a singleton this theory is to be found, in its dual form, in a recent paper of Ehresmann [3].

I would Iike to thank I. Connell for some interesting conversations on rings during the preparation of this note.

2. Weak families. Let $\underline{A}$ and $E$ be two categories and denote by $F: \underline{A} \rightarrow \underline{E}$ a covariant functor.

A family $\left(\mathrm{a}_{\alpha}\right)$ of morphisms $\mathrm{a}_{\alpha}: \mathrm{A} \rightarrow \mathrm{A}_{\alpha}$ of $\underline{\mathrm{A}}$ will be called an $F$-weak family or a weak family if it has the following property: if a morphism $f: \overline{F(B) \rightarrow F(A)}$ is such that there exists a family ( $b_{\alpha}$ ) of morphisms $b_{\alpha}: B \rightarrow A_{\alpha}$ with, for each $\alpha, F\left(a_{\alpha}\right) \circ f=F\left(b_{\alpha}\right)$, then there exists a unique $\mathrm{b}: \mathrm{B} \rightarrow \mathrm{A}$ with $F(b)=f$ and, for each $\alpha, a_{\alpha} \circ b=b_{\alpha}$. A morphism a : $A \rightarrow C$ of $A$ is called weak if it is a weak family when viewed as a family indexed by a one-point set. In the terminology of Ehresmann [3], a weak morphism is an (E, F) injection.

$$
\text { If (a }{ }_{\alpha} \text { ) is a family of morphisms } a_{\alpha}: A \rightarrow A_{\alpha} \text { the }
$$
family ( $A_{\alpha}$ ) will be called the range of the family and the object $A$ will be referred to as its domain. Two families $\left(a_{\alpha}\right)$ and ( $\left.{ }^{\prime}{ }_{\alpha}\right)$, with the same range and with domains $A$ and $A^{\prime}$, will be called isomorphic if there is an isomorphism a of $A$ with, for each $\alpha, \mathrm{a}_{\alpha} \circ \mathrm{a}=\mathrm{a}^{\prime}{ }_{\alpha}$.

\section{Examples.}

1. A family $\left(a_{\alpha}\right)$ of morphisms of $A$ will be said to have the left cancellation property (LCP) if $b=c$ whenever, for each $\alpha, a_{\alpha} \circ b=a_{\alpha} \circ c$. When $\underline{A}=\underline{E}$ and $F$ is the identity functor it follows that $\left(\mathrm{a}_{\alpha}\right)$ is weak if and only if (a $\left.{ }_{\alpha}\right)$ has the LCP. In particular, a morphism is weak if and only if 
it is a monomorphism. Consequently, a weak family can be thought of as a generalized monomorphism.

2. Let $A$ be the category of uniform spaces, $E$ be the category of sets, and let $F$ be the forgetful functor. Then, a family $\left(a_{\alpha}\right)$ of uniformly continuous functions is weak if and only if the uniformity on $F(A), A$ being the domain, is the weak uniformity defined by the functions $F\left(a_{\alpha}\right)$ and the uniformities $\underline{\mathrm{U}}_{\alpha}$ on the sets $\mathrm{F}\left(\mathrm{A}_{\alpha}\right)$.

3. Let $E$ be the category with a unique morphism. There is a unique functor $F: \underline{A} \rightarrow \underline{E}$. A family $\left(a_{\alpha}\right)$ of morphisms $a_{\alpha}$ is weak if and only if $\left(A,\left(a_{\alpha}\right)\right)$ is a direct product of the family ( $\mathrm{A}_{\alpha}$ ). Hence, weak families might well be called relative direct products.

4. Let $A$ be the category of groups. Take $F$ to be the forgetful functor from $A$ to the category of sets. A family $\left(a_{\alpha}\right.$ ) of group homomorphisms $\dot{a}_{\alpha}: A \rightarrow A_{\alpha}$ is $F$-weak if and only if $\bigcap_{\alpha} \operatorname{ker}\left(a_{\alpha}\right)$ is the trivial subgroup of $A$. In particular a group homomorphism is weak if and only if it is a monomorphism.

5. Let A denote the category of vector spaces over the field of rationals. For $\underline{E}$ take the category of abelian groups, and Iet $F(A)$ denote the underlying abelian group of $A$. Then, every family of linear transformations $a_{\alpha}: A \rightarrow A_{\alpha}$ is weak.

6. Let $A$ denote a ring $R$, with unit, viewed as a category with one object 1 and morphisms the elements of the ring, the law of composition being ring multiplication. A left $R$-module defines a covariant functor $M: \underline{A} \rightarrow \underline{E}$ where $\underline{E}$ is the category of abelian groups.

A ring element $r$ is $M$-weak if, for a group homomorphism $f, r \cdot f(x)=s \cdot x$ for all $x \in M(1)$ implies that there exists a unique $t \in R$ with $f(x)=t \cdot x$ for all $x \in M(1)$. In order that $M$-weak elements exist, it is necessary that $M$ have zero annihilator. Clearly, an element of the ring with a left inverse is M-weak for all such modules $M$. 
Conversely, if $r$ is $M$-weak for all modules $M$ with zero annihilator then $r$ is left-invertible. Consider the left $R$-module $M=R \times R /(r)$, where $(r)$ is the principal left ideal determined by $r$. Define $f: M \rightarrow M$ by $f(x, y)=(0, y)$. Then $r \cdot f(x, y)=0 \cdot(x, y)=0$. The group homomorphism $f$ is of the form $f(x, y)=t \cdot(x, y)$ if and only if $R /(r)=\{0\}$. This is equivalent to $r$ being left-invertible.

Every ring $\mathrm{R}$ is a left $\mathrm{R}$-module with zero annihilator. The element 0 is $R$-weak if and only if every endomorphism $f$ of the additive group of $R$ is given by left multiplication with some element of the ring. For example, 0 is a $\mathbb{Z}$-weak element of $\mathbb{Z}$.

7. Let $A$ again denote a ring $R$ viewed as a category, and let $E$ now be the category of sets. Let $F$ be the composition of the functor corresponding to $R$ as a left $R$-mcilule with the forgetful functor from the category of groups to $E$.

A ring element $r$ is $F$-weak if, for a function $f$, $r \cdot f(x)=s \cdot x$ for all $x \in F(1)$ implies that there exists $t \in R$ with $f(x)=t \cdot x$ for all $x \in F(1)$. When $0 \neq 1$ this is equivalent to $r$ not being a left divisor of zero.

Assume $r \in R$ is not a left divisor of zero. Let $f: R \rightarrow R$ be a function for which there exists $s$ with $r \cdot f(x)=s \cdot x$ for aII $x \in R$. Let $t=f(1)$. Then, $r \cdot f(x)=(r \cdot t) \cdot x$, for aII $x \in R$. Since $r$ is not a left divisor of zero, $f(x)=t \cdot x$ for all $x \in r$. In other words, $r$ is $F$-weak.

Assume that $r$ is $F$-weak. Then $r \neq 0$. Let $r$ be a Ieft divisor of zero and let $p \in R$ be such that $r \cdot p=0$ and $p \neq 0$. Define $f: R \rightarrow R$ by $f(x)=0$ if $x \neq p$ and $f(p)=p$. Then, $r \cdot f(x)=0 \cdot x$ for all $x \in R$. Hence, there exists $t \in R$ with $f(x)=t \cdot x$ for all $x \in R$. Since $1 \neq p, 0=f(1)=t$. This is a contradiction.

3. Elementary properties of weak families. As might be expected, a weak family $\left(a_{\alpha}\right)$ is determined up to isomorphism by its range and $\left(F\left(a_{\alpha}\right)\right)$. 
PROPOSITION 1. Let $\left(a_{\alpha}\right)$ and $\left(a_{\alpha}{ }^{\prime}\right)$ be two weak families with the same range. They are isomorphic if, for each $\alpha, \quad F\left(a_{\alpha}\right)=F\left(a_{\alpha}{ }^{\prime}\right)$.

Proof: If $A$ and $A^{\prime}$ are the respective domains of $\left(a_{\alpha}\right)$ and $\left(a_{\alpha}^{\prime}\right)$, then $F(A)=F\left(A^{\prime}\right)=X$. Therefore, $F\left(a_{\alpha}\right) \circ 1_{X}=F\left(a_{\alpha}{ }^{\prime}\right)=F\left(a_{\alpha}{ }^{\prime}\right) \circ 1_{X}=F\left(a_{\alpha}\right)$. It follows that there are unique morphisms $a: A^{\prime} \rightarrow A$ and $a^{\prime}: A \rightarrow A^{\prime}$ such that, for each $\alpha, a_{\alpha} \circ a=a_{\alpha}{ }^{\prime}$ and $a_{\alpha}{ }^{\prime} \circ a^{\prime}=a_{\alpha^{\prime}}$ and $F(a)=F\left(a^{\prime}\right)$ $=1 \mathrm{X}$. The uniqueness condition in the definition of a weak family implies that $a$ and $a^{\prime}$ are inverse to one another.

A family ( $a_{\alpha}$ ) of morphisms will be said to have the left cancellation property ( $L$ C P) if, for each $\alpha, a_{\alpha} \circ b=a_{\alpha} \circ \mathrm{c}$ implies $b=c$. If the family $\left(a_{\alpha}\right)$ defines a direct product in A of the family $\left(A_{\alpha}\right)$ of objects $A_{\alpha}$ of $\underline{A}$, then $\left(a_{\alpha}\right)$ has the L C P.

In general, if ( $\left.\mathrm{A}_{\alpha}\right)$ has the L C P the family $\left(F\left(a_{\alpha}\right)\right)$ need not have this property. However, when $F$ has a left adjoint the family $\left(F\left(a_{\alpha}\right)\right)$ inherits the LCP from ( $\left.a_{\alpha}\right)$.

Since a weak family can be thought of as a generalized or relative direct product, the question arises as to whether a family $\left(a_{\alpha}\right)$ that defines a direct product in $\underline{A}$ is weak.

PROPOSITION 2. Let $\left(a_{\alpha}\right)$ define a direct product in A. The following are equivalent:

(1) $\left(a_{\alpha}\right)$ is weak;

(2) for each family $\left(\mathrm{b}_{\alpha}\right)$ of morphisms of $A$, with domain $B$ and range $\left(A_{\alpha}\right)$, there is a unique morphism $f: F(B) \rightarrow F(A)$ with, for each $\alpha, F\left(a_{\alpha}\right) \circ f=F\left(b_{\alpha}\right)$. 
In particular, $\left(a_{\alpha}\right)$ is weak if $F$ has a left adjoint or, more generally, if $\left(F\left(a_{\alpha}\right)\right)$ has the L C P.

Proof: Since $\left(a_{\alpha}\right)$ defines a direct product in $A$, for each family $\left(b_{\alpha}\right)$ of morphisms of $\underline{A}$, with domain $B$ and range $\left(\mathrm{A}_{\alpha}\right)$, there is a unique $\mathrm{b}: \mathrm{B} \rightarrow \mathrm{A}$ with, for each $\alpha$, $\mathrm{a}_{\alpha} \circ \mathrm{b}=\mathrm{b}_{\alpha}$. Consequently, there is at most one $f: F(B) \rightarrow F(A)$ of the form $F(b)$ where $b$ satisfies, for each $\alpha, a_{\alpha} \circ b=b_{\alpha}$. From this observation, it follows immediately that (1) and (2) are equivalent.

\section{Examples.}

8. Let both $A$ and $E$ be the category of topological spaces and let $F$ be the functor defined by the generalized Stone-Cech compactification. It is well known that $F$ does not preserve direct products [4]. However, every family (a $\left.{ }_{\alpha}\right)$ of continuous functions that defines a direct product is $F$-weak.

9. Let $A=E$ be the category of abelian groups and let $F$ be the functor obtained by associating with each group $A$ the tensor product $A \otimes \mathbb{Q}$. Denote by $A$ a direct product of the modules $\mathbb{Z}_{i}$, where $i=1,2,3, \ldots$ and by $\left(a_{i}\right)$ the family of projections $a_{i}: A \rightarrow \mathbb{Z}_{i}$. The family $\left(a_{i}\right)$ is not $F$-weak. Clearly, for each $i, F\left(\mathbb{Z}_{i}\right)=\mathbb{Z}_{i} \otimes \mathbb{Q}$ is the zero group and $F(A)=A \otimes \mathbb{Q}$ is not the zero group. Hence, there are at least two morphisms $f_{1}, f_{2}: F(A) \rightarrow F(A)$ with, for each $i$, $F\left(a_{i}\right) \circ f_{j}=F\left(a_{i}\right)=0$.

The following proposition is a converse to proposition 2.

PROPOSITION 3. If $\left(a_{\alpha}\right)$ is a weak family in $A$ for which the family $\left(F\left(a_{\alpha}\right)\right)$ defines a direct product of the family $\left(F\left(A_{\alpha}\right)\right)$, then $\left(a_{\alpha}\right)$ defines a direct product of the family $\left(A_{\alpha}\right)$. 
Proof: Let $\left(b_{\alpha}\right)$ be a family of morphisms $b_{\alpha}: B \rightarrow A_{\alpha}$ of $A$. There is a unique map $f: F(B) \rightarrow F(A)=X$ with, for each $\alpha, f_{\alpha} \circ f=F\left(b_{\alpha}\right)$. Since $\left(a_{\alpha}\right)$ is weak and $F\left(a_{\alpha}\right)=f_{\alpha}$, there is a unique map $\mathrm{b}: \mathrm{B} \rightarrow \mathrm{A}$ with, for each $\alpha, \mathrm{a}_{\alpha} \circ \mathrm{b}=\mathrm{b}_{\alpha}$. Hence, $\left(a_{\alpha}\right)$ defines a direct product of $\left(\mathrm{A}_{\alpha}\right)$.

Let $B$ be a third category and let $F: \underline{A} \rightarrow \underline{E}$ be equal to $H G$, where $G: \underline{A} \rightarrow \underline{B}$ and $H: \underline{B} \rightarrow \underline{E}$.

PROPOSITION 4. When $H$ is faithful, a family $\left(a_{\alpha}\right)$ of morphisms of $A$ is G-weak if it is F-weak. If the family of morphisms $\left(a{ }_{\alpha}\right)$ is G-weak and the family $\left(G\left(a_{\alpha}\right)\right)$ is H-weak, then $\left(a_{\alpha}\right)$ is F-weak.

Proof: Assume that $\left(b_{\alpha}\right)$ is a family of morphisms $b_{\alpha}: B \rightarrow A_{\alpha}$ and that $g: G(B) \rightarrow G(A)$ is such that, for each $\alpha$, $\mathrm{G}\left(\mathrm{a}_{\alpha}\right) \circ \mathrm{g}=\mathrm{G}\left(\mathrm{b}_{\alpha}\right)$. Then, for each $\alpha, F\left(\mathrm{a}_{\alpha}\right) \circ \mathrm{H}(\mathrm{g})=\mathrm{F}\left(\mathrm{b}_{\alpha}\right)$. Consequently, there is a unique $\mathrm{b}: \mathrm{B} \rightarrow \mathrm{A}$ with $\mathrm{F}(\mathrm{b})=\mathrm{H}(\mathrm{g})$ and, for each $\alpha, a_{\alpha} \circ b=b_{\alpha}$.

Since $F(b)=H G(b)=H(g)$, the faithfulness of $H$ implies that $g=G(b)$. Clearly, there is at most one $b$ with $G(b)=g$ and satisfying the condition $a_{\alpha} \circ b=b_{\alpha}$ for each $\alpha$.

Let $\left(b_{\alpha}\right)$ be a family of morphisms $b_{\alpha}: B \rightarrow A_{\alpha}$ and let $f: F(B) \rightarrow F(A)$ be such that, for each $\alpha, F\left(a_{\alpha}\right) \circ f=F\left(b_{\alpha}\right)$. Since $\left(G\left(a_{\alpha}\right)\right)$ is H-weak, there is a unique $g: G(B) \rightarrow G(A)$ with, for each $\alpha, G\left(a_{\alpha}\right) \circ g=G\left(b_{\alpha}\right)$ and $H(g)=f$. The G-weakness of $\left(a_{\alpha}\right)$ implies that there exists a unique $b: B \rightarrow A$ with, for each $\alpha, a_{\alpha} \circ b=b_{\alpha}$ and $G(b)=g$.

Clearly, $F(b)=f$. It remains to show the uniqueness of $b$. Let $b^{\prime}: B \rightarrow A$ be such that, for each $\alpha, a_{\alpha} \circ b^{\prime}=b_{\alpha}$ and 
$F\left(b^{\prime}\right)=f$. Then, $G\left(b^{\prime}\right)=g$ since, for each $\alpha$, $G\left(a_{\alpha}\right) \circ G\left(b^{\prime}\right)=G\left(b_{\alpha}\right)$ and $H G\left(b^{\prime}\right)=F\left(b^{\prime}\right)=f$. It then follows from the G-weakness of $\left(a_{\alpha}\right)$ that $b^{\prime}=b$.

\section{Examples.}

10. Let $A$ be a ring $R$ viewed a s category and let $\underline{B}$ denote the category of abelian groups. Let $G: \underline{A} \rightarrow \underline{B}$ be the functor corresponding to $R$ as a left $R$-module and let $H: \underline{B} \rightarrow \underline{E}$, where $\underline{E}$ is the category of sets, be the forgetful functor. Then, $F=\overline{H G}$ is the functor of example 7. The faithfulness of $\mathrm{H}$ and proposition 4 imply that every $r \in R$ which is not a divisor of zero is G-weak.

11. Let $\underline{A}=\underline{B}$ be the category of abelian groups and let $E$ be the trivial category with one morphism. Denote by $G$ the functor of example 9 and by $H$ the unique functor from $B$ to $\underline{E}$. The family $\left(a_{i}\right)$ of example 9 is then $F=H G$-weak, but it is not G-weak.

4. Weak families and direct products. Let $\Omega$ be an index set, and for each $\alpha \in \Omega$ let $I(\alpha)$ be an index set. For each $\beta \in I(\alpha)$ let $a_{\alpha \beta}^{\prime}: A_{\alpha} \rightarrow A_{\alpha \beta}$ be a morphism of $A$, and for each $\alpha \in \Omega$ let $\mathrm{a}_{\alpha}: \mathrm{A} \rightarrow \mathrm{A}_{\alpha}$. Define $\mathrm{a}_{\alpha \beta}$ to be $\mathrm{a}_{\alpha \beta}^{\prime} \circ \mathrm{a}_{\alpha}$.

PROPOSITION 5. If $\left(a_{\alpha}\right)$ and, for each $\alpha$, $\left(a_{\alpha \beta}^{\prime}\right)$ are weak families, the family $\left(a_{\alpha \beta}\right)$ is weak. Conversely, if $\left(\mathrm{a}_{\alpha \beta}\right)$ is weak the family $\left(\mathrm{a}_{\alpha}\right)$ is weak.

Proof: Let $\left(\mathrm{b}_{\alpha \beta}\right)$ be a family of morphisms $\mathrm{b}_{\alpha \beta}: \mathrm{B} \rightarrow \mathrm{A}_{\alpha \beta}$. Assume $f: F(B) \rightarrow F(A)$ is such that, for each $\alpha$ and $\beta$, $F\left(a_{\alpha \beta}\right) \circ f=F\left(b_{\alpha \beta}\right)$.

Let $f_{\alpha}: F(B) \rightarrow F\left(A_{\alpha}\right)$ be the morphism $F\left(a_{\alpha}\right) \circ f$. Since, for each $\alpha,\left(a_{\alpha \beta}^{\prime}\right)$ is weak, there is, for each $\alpha$, a unique morphism $b_{\alpha}: B \rightarrow A_{\alpha}$ with $F\left(b_{\alpha}\right)=f_{\alpha}$ and, for each $\beta$, $a_{\alpha \beta}^{\prime} \circ b_{\alpha}=b_{\alpha \beta}$. 
Since $f_{\alpha}=F\left(a_{\alpha}\right) \circ f=F\left(b_{\alpha}\right)$, there is a unique $b: B \rightarrow A$ with $F(b)=f$ and, for each $\alpha, a_{\alpha} \circ b=b_{\alpha}$. It remains to show that $b$ is the unique morphism with $F(b)=f$ and, for each $\alpha$ and $\beta, a_{\alpha \beta} \circ b_{\alpha \beta}$.

Assume $F\left(b^{\prime}\right)=f$ and that, for each $\alpha$ and $\beta$, $a_{\alpha \beta} \circ b^{\prime}=b_{\alpha \beta}$. Let $b_{\alpha}^{\prime}=a_{\alpha} \circ b^{\prime}$. Then, $\quad F\left(b_{\alpha}^{\prime}\right)=F\left(a_{\alpha}\right) \circ f=f_{\alpha}$ and, for each $\beta, a_{\alpha \beta}^{\prime} \circ b_{\alpha}^{\prime}=b_{\alpha \beta}$. Therefore, $b_{\alpha}^{\prime}=b_{\alpha}$. From this it follows immediately that $b=b^{\prime}$.

The proof of the converse is similar.

A morphism $e$ of $A$ will be called an embedding if $e$ is. weak and $F(e)$ is a monomorphism. An object $A$ of $A$ will be called a subobject of $B$ if there is an embedding $e: A \rightarrow B$.

PROPOSITION 6. Let $\left(A_{\alpha}\right)$ be a family of objects $A_{\alpha}$ of $A$. Let $\left(\pi A_{\alpha}, \mathrm{pr}_{\alpha}\right)$ be a direct product of the family $\left(\mathrm{A}_{\alpha}\right)$. Assume that $\left(\mathrm{pr}_{\alpha}\right)$ is weak and that $\left(\mathrm{F}\left(\mathrm{pr}_{\alpha}\right)\right)$ has the $\mathrm{L}$ C P. The following statements are equivalent:

(1) $A$ is a subobject of $\Pi A_{\alpha}$;

(2) there is a weak family $\left(a_{\alpha}\right)$ with domain $A$ and range $\left(\mathrm{A}_{\alpha}\right)$ for which $\left(\mathrm{F}\left(\mathrm{a}_{\alpha}\right)\right)$ has the L C P.

Proof: There is a 1-1 correspondence between families ( ${ }_{\alpha}$ ) with domain $A$ and range $\left(A_{\alpha}\right.$ ) and morphisms $e: A \rightarrow \Pi A_{\alpha}$. To the morphism e corresponds the family $\left(a_{\alpha}\right)$ where, for each $\alpha, a_{\alpha}=p r_{\alpha} \circ \mathrm{e}$. Since $\left(\mathrm{pr}_{\alpha}\right)$ is weak, proposition 5 shows that $e$ is weak if and only if the corresponding family $\left(a_{\alpha}\right)$ is weak.

If $\left(F\left(a_{\alpha}\right)\right)$ has the $L C P$ it is clear that $F(e)$ is a monomorphism. Conversely, since $\left(F\left(\mathrm{pr}_{\alpha}\right)\right)$ has the $\mathrm{L} C \mathrm{P}$, 
the family $\left(F\left(a_{\alpha}\right)\right)$ has the LCP whenever $F(e)$ is a monomorphism.

\section{Example.}

12. Let $A$ be the category of topological spaces, let $\underline{E}$ be the category of sets, and let $F$ be the forgetful functor. A family ( $\left.a_{\alpha}\right)$ with domain $A$ is such that $\left(F\left(a_{\alpha}\right)\right)$ has the L C P if and only if the functions $a_{\alpha}$ separate the points of $F(A)$. Hence, the embedding Iemma in [5] is a particular case of the result in proposition 6. This proposition also shows that a similar embedding lemma holds for uniform spaces.

\section{REFERENCES}

1. N. Bourbaki, Topologie Générale, Actualités Sci. Ind. 1142 3éme ed., 1961.

2. , Théorie des Ensembles, Actualités Sci. Ind. 1258,1957 .

3. D. Ehresmann, Structures Quotient, Comm. Math. Helvetici, vol. 38 (1964), 219-242.

4. L. Gillman and M. Jerison, Rings of Continuous Functions, New York, 1960.

5. J. L. Kelley, GeneraI Topology, New York, 1955.

\section{McGill University}

